\title{
Impairment of microcirculation in juvenile idiopathic arthritis - studies by nailfold videocapillaroscopy and correlation with serum levels of SICAM and VEGF
}

\author{
Anna Gorska1,3, Otylia Kowal-Bielecka ${ }^{2}$, Miroslawa Urban'1, Slawomir Chlabicz ${ }^{3}$, \\ Jerzy Sienkiewicz ${ }^{4}$, Stanislaw Gorski ${ }^{3}$
}

12nd Department of Children's Diseases, 2 Department of Rheumatology and Internal Medicine, ${ }^{3}$ Department of Family Medicine and Community Nursing, ${ }^{4}$ Department of Statistics and Medical Informatics, Medical University of Bialystok, Bialystok, Poland

\begin{abstract}
Impairment of vascular endothelium plays a key role in the pathogenesis of inflammatory diseases including juvenile idiopathic arthritis (JIA) and atherosclerosis. We hypothesized that structural abnormalities of the smallest blood vessels (capillaries) might exist and reflect endothelial dysfunction in children with JIA. Microcirculation was studied, by means of nailfold videocapillaroscopy with computer-associated image analysis, in 43 patients with JIA and compared with 20 healthy children. Moreover, capillaroscopic findings were correlated with the activity of the disease and the levels of serum biomarkers of endothelial injury, namely soluble intercellular adhesion molecule (sICAM) and vascular endothelial growth factor (VEGF). We found that in JIA patients capillaries were significantly wider and longer than in healthy controls. Moreover, irregular capillaries and dilated subpapillary venous plexus were found significantly more frequently in JIA in comparison with the control group. Serum levels of sICAM and VEGF were significantly higher in JIA patients with capillary abnormalities than in JIA patients with normal capillaroscopy. Our study indicates that there are structural changes in the microcirculation of patients with JIA and that these changes might reflect endothelial injury. Whether capillaroscopy might have a role in early identification of JIA patients being at higher risk of atherosclerosis requires further studies.
\end{abstract}

Key words: juvenile idiopathic arthritis, mircrocirculation, capillaroscopy

\section{Introduction}

Activation of vascular endothelium is considered to play a key role in the initiation and progression of systemic inflammatory diseases such as rheumatoid arthritis (RA) and juvenile idiopathic arthritis (JIA) [1-3]. Recently, chronic inflammation has also been recognized as a pivotal factor in the pathogenesis of atherosclerosis. Indeed, recent data indicate that the atherosclerotic process in autoimmune diseases, including JIA and RA, progresses concomitantly and increases the risk of cardiovascular disease by up to 2-5 times [4-7].

Capillaroscopy is an non-invasive technique which allows in vivo investigation of the smallest blood vessels [8-10].

Different capillaroscopic abnormalities have been shown to occur in several systemic autoimmune dis-

Correspondence: A. Górska, 15-101 Białystok,

ul Jurowiecka 28/4, Poland; e-mail: agorska50@wp.pl eases, such as systemic sclerosis, dermatomyostis, mixed connective tissue disease and RA [11-13]. However, so far only very little is known regarding capillaroscopy findings in JIA [14,15].

Taking into account the role which endothelial injury is considered to play in the pathogenesis of systemic inflammatory diseases including JIA and atherosclerosis we hypothesised that structural abnormalities of the smallest blood vessels (capillaries) may occur in children with JIA and might reflect endothelial injury due to systemic inflammation [16-18].

To investigate this hypothesis videocapillaroscopy with computerized image analysis of the skin microcirculation was performed in a cohort of children with JIA in comparison with age- and sex-matched healthy children. Moreover, capillaroscopic findings were correlated with the activity of the disease. To evaluate potential relationships between structural abnormalities in microcirculation and impairment of vascular endothelium, we investigated associations between 
Table 1. Clinical characteristics and the results of capillaroscopic measurements in patients with JIA and healthy controls.

\begin{tabular}{|c|c|c|c|c|}
\hline Parameters & Total study group & 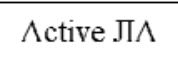 & Inactive Л $\Lambda$ & Control group \\
\hline Number(Boys/Girls) & $43(23 / 20)$ & $25(14 / 11)$ & $18(9 / 9)$ & $20(11 / 9)$ \\
\hline$\Lambda g e$ (years) & $10.6 \pm 4.3$ & $9.8 \pm 4.1$ & $11.8 \pm 4.3$ & $10.9 \pm 3.8$ \\
\hline Disease duration (years) & $3.7 \pm 3.4$ & $4.5 \pm 3.7$ & $2.7 \pm 2.5$ & - \\
\hline $\mathrm{CRP}(\mathrm{mg} / \mathrm{dl})$ & $1.4 \pm 1.5$ & $2.2 \pm 1.5$ & $0.3 \pm 0.4$ & \\
\hline $\operatorname{PLT}\left(10^{3} / \mathrm{ml}\right)$ & & $377.5 \pm 110.4$ & $270.4 \pm 56.5$ & \\
\hline Presence of dilated subpapillary venous plexus n (\%) & $20(45.9)^{*}$ & $12(47.8)^{*}$ & $8(42.9)^{*}$ & $4(20.0)$ \\
\hline Presence of irregular loops n (\%) & $26(59.4)^{* *}$ & $17(69.4)^{* *}$ & $8(42.8)^{* *}$ & $2(10.0)$ \\
\hline Capillaries density $/ \mathrm{mm}^{2}$ & $14.1 \pm 4.2$ & $13.1 \pm 3.9$ & $15.4 \pm 4.3$ & $16.2 \pm 6.1$ \\
\hline I.ength loops (um) & $182.0 \pm 73.1^{*}$ & $189.9 \pm 81.5^{*}$ & $170.2 \pm 58.8^{*}$ & $139.6 \pm 52.6$ \\
\hline Width of arterial limbs (um) & $35.4 \pm 11.4 * *$ & $35.6 \pm 11.6^{* * *}$ & $34.8 \pm 11.2^{* * *}$ & $24.7 \pm 9.1$ \\
\hline Width of venous limbs (um) & $45.3 \pm 26.5^{* *}$ & $47.6 \pm 32.1 \%$ & $41.8 \pm 14.7^{* * *}$ & $27.0 \pm 8.2$ \\
\hline
\end{tabular}

Data are presented as means $\pm \mathrm{SD} ; * \mathrm{p}<0.05$ compared with control group; ** $\mathrm{p}<0,01$ compared with control group.

abnormal capillaroscopic findings and serum levels of soluble intercellular adhesion molecule (sICAM) and vascular endothelial growth factor (VEGF) which are considered to be biomarkers of vascular injury in chronic inflammatory conditions such as RA, JIA and atherosclerosis.

\section{Materials and methods}

Patients. Forty three children, aged 4-17 years (mean 10.6, S.D. 4.3), with JIA diagnosed according to ILAR (International League Against Rheumatism) criteria [19] were included. JIA patients with signs of infections or any known risk factors of atherosclerosis except JIA were excluded. Nineteen had polyarticular course JIA (6 with systemic onset with polyarticular course, 5 with oligoarticular onset and extension to a polyarticular course) and 25 had a persistent oligoarticular course. Disease was considered active if active joint inflammation was detected at physical examination in at least one joint (swelling or tenderness if swelling was not present), and at least one of the following parameters was present: erythrocyte sedimentation rate $(\mathrm{ESR})>15 \mathrm{~mm}$ after $1^{\text {st }}$ hour, C-reactive protein (CRP) over $0.5 \mathrm{mg} / \mathrm{dL}$, and/or platelet count (PLT) over $300 \times 10^{3} / \mathrm{mL}$. These criteria are in agreement with those used in previous studies [20].

39 children were treated with disease modifying antirheumatic drugs (DMARDs). These included: salazopyrin (SN) in 9 patients, methotrexate (MTX) in 28, or etanercept in 11 patients. 25 patients with active disease were receiving low dose corticosteroids (less than or equal to $5 \mathrm{mg}$ of prednisolone/day orally), and 13 of them were taking non-steroidal anti-inflammatory drugs (NSAIDs) also. Five children with inactive disease did not receive any medications.

The control group comprised 20 healthy children of matched age, without history of infectious diseases within last four weeks. Clinical characteristics of the JIA patients and the control group is presented in Table 1.

Capillaroscopy. Capillaroscopic examination was performed by means of Video Capillaroscope (SCALAR Corporation, Japan) using 200x magnification, after 20 minutes of acclimatization of a patient to a constant temperature of $20-22^{\circ} \mathrm{C}$. Using frame regis- tration software, digitized video images from the microscope were combined to form a panoramic mosaic of the nailfold. Fingers II$\mathrm{V}$ of both hands were assessed with respect of the morphology of loops (irregular/tortuous capillaries) as well as the presence of dilated subpapillary venous plexus. Capillaroscopy was considered abnormal if the number of tortuous and/or irregular capillaries exceeded 2 per finger and the changes were present in at least two fingers out of eight fingers investigated. Detailed measurements were performed on the central part of the left fourth finger nailfold and included evaluation of capillaries density per $1 \mathrm{~mm}^{2}$, and the width of both arterial and venous arms (the width of a column of red blood cells). Capillaroscopy images were captured, coded, stored, and subsequently evaluated by one researcher with a longstanding experience in capillaroscopy (AG) in a blinded fashion.

Blood for analysis was collected on an empty stomach, in the morning hours. The sera obtained after centrifugation were stored at a temperature of $-70^{\circ} \mathrm{C}$. Serum levels of sICAM-1 and VEGF were determined using commercially available Enzyme-Linked Immunosorbent Assay (ELISA) Quantikine kits (R\&D Systems, USA) according to the manufacturer's instructions. The lowest detectable concentrations of sICAM-1 and VEGF were: $0.35 \mathrm{ng} / \mathrm{mL}$ and $9.0 \mathrm{pg} / \mathrm{mL}$, respectively.

Ethical issues. The study protocol was approved by the Local Bioethics Committee.

Statistical analysis. Statistical analysis was performed with the use of the computer program "Statistica 6.0". To compare study groups with the control group, the Student's unpaired t-test was used for normally distributed variables and the Mann-Whitney U test for non-normally distributed variables. Correlations were analyzed by Pearson or Spearman method as appropriate. The data are presented as means and standard deviation (S.D.) or range. A p value $<0.05$ was considered to be statistically significant.

\section{Results}

\section{Capillaroscopy}

Capillaroscopic measurements are presented in Table 1. Mean number of capillaries in JIA patients was 


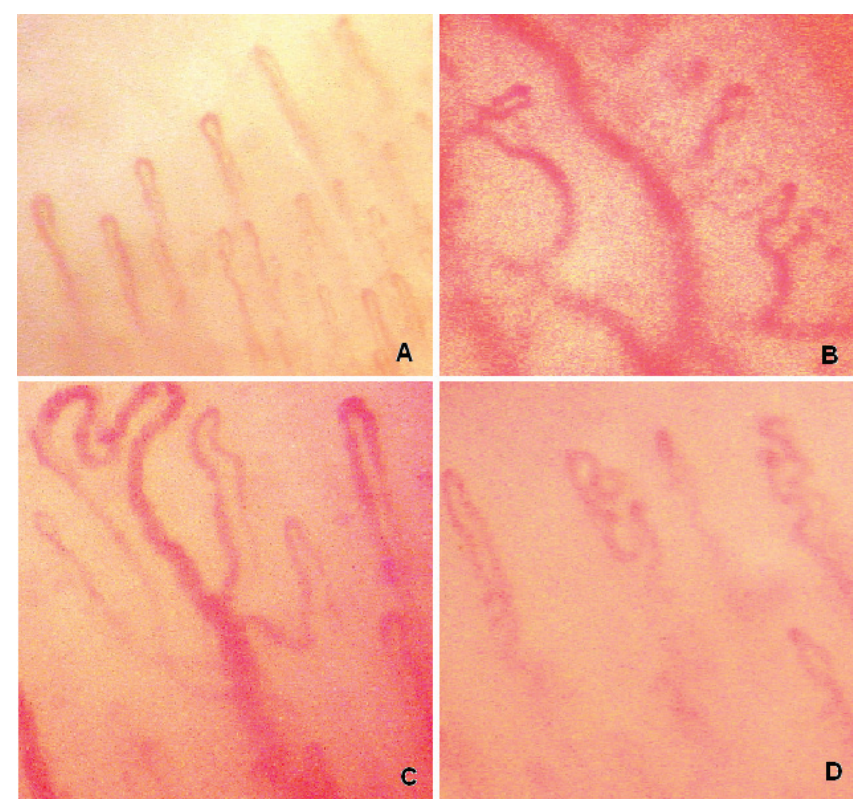

Fig. 1. Capillaroscopic pictures of (A) normal capillary loops, (B) dilated subpapillary venous plexus, and $(\mathbf{C}$ and $\mathbf{D})$ irregular/tortuous loops (original magnification $\times 100-\mathrm{A}$ or $\times 200-\mathrm{B}$-D).

$14.1 / \mathrm{mm}^{2}$ (S.D. 4.2, range 5-23) and did not differ significantly from the number in healthy children: $16.3 / \mathrm{mm}^{2}$ (S.D. 6.1, range 7-33).

The capillaries were significantly longer in JIA patients when compared with age-matched healthy controls: their average length was $182.3 \mu \mathrm{m}$, (S.D. 73.2, range 79.2-442.9) in JIA patients vs. $139.6 \mu \mathrm{m}$ (S.D. 52.6, range 64.1-287.0) in healthy children $(p<0.01)$. Also the width of arterial and venous limbs of capillaries in JIA compared to healthy children differed significantly: $35.4 \mu \mathrm{m}$ (S.D. 11.4, range 10.8$55.8)$ vs. $24.7 \mu \mathrm{m}$ (S.D. 9.1, range 12.9-46.3) $(\mathrm{p}<0.001)$ for arterial limb and $45.3 \mu \mathrm{m}$ (S.D. 26.5, range 14.07-78.00) vs. $27.1 \mu \mathrm{m}$ (S.D. 8.2, range 14.846.7) $(\mathrm{p}<0.001)$ for venous limb.

Dilated subpapillary venous plexus and irregular loops (Fig. 1) were present in $45.9 \%$ and $59,4 \%$ patients in total study group JIA respectively, and in 20 $\%$ and $10 \%$ of healthy controls respectively $(\mathrm{p}<0.05$, $\mathrm{p}<0.01$ vs JIA for both: venous plexus and irregular loops).

Active JIA was diagnosed in $25(58.1 \%)$ children, inactive JIA - in $18(41.9 \%)$ children. The groups did not differ with respect to age or sex.

There were no significant differences between active and inactive JIA subgroups with respect to capillary density, length or width of capillary loops or the presence of abnormal findings - Table 1 .

\section{SICAM and VEGF}

Mean serum concentration of sICAM-1 in active JIA patients was significantly higher $(333.44 \mathrm{ng} / \mathrm{mL}$, S.D.

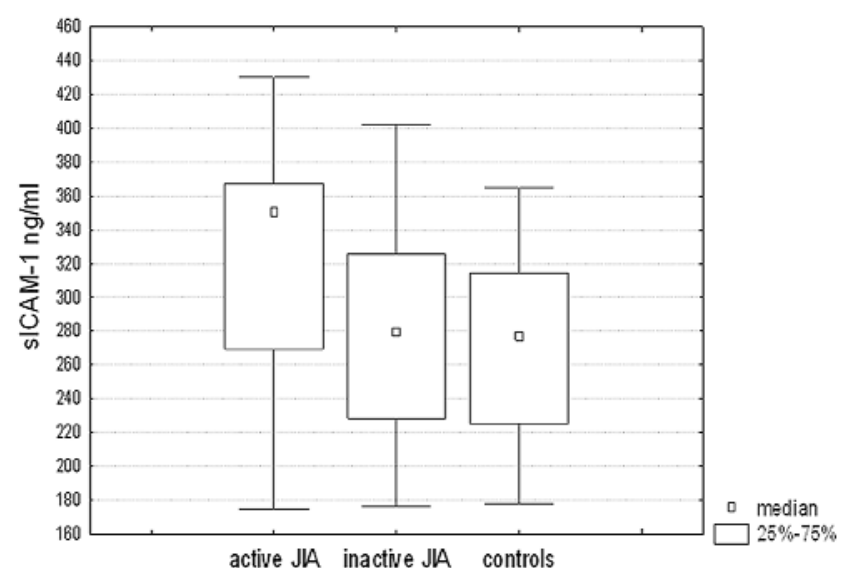

Fig. 2. Mean serum levels of soluble intercellular adhesion molecule-1 (sICAM-1) in active JIA patients compared to inactive JIA patients $(\mathrm{p}<0.05)$ and healthy controls $(\mathrm{p}<0.05)$.

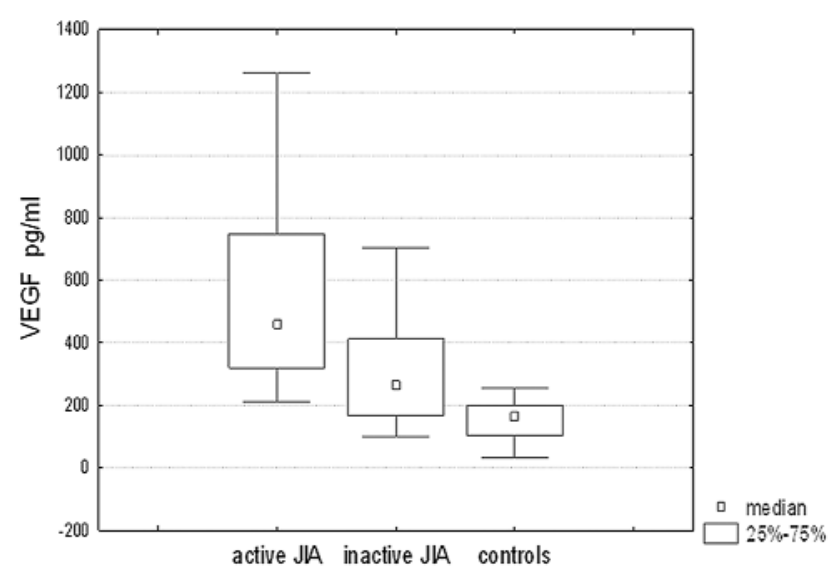

Fig. 3. Mean serum levels of vascular endothelial growth factor (VEGF) in active JIA patients compared to inactive JIA patients $(p<0.001)$ and healthy controls $(p<0.001)$.

109.9, range 194.6-728.9) than mean levels in inactive JIA patients $(276.53 \mathrm{ng} / \mathrm{mL}$, S.D. 67.5, range $174.5-$ $402.1, \mathrm{p}<0.05$ vs active) and healthy controls ( 288.66 ng/mL, S.D. 102.4, range 177.3-662.6, $\mathrm{p}<0.05$ vs active). No significant differences between inactive JIA subgroup and healthy control were detected (Fig. 2)

Mean serum concentration of VEGF in active JIA patients $(615.58 \mathrm{pg} / \mathrm{mL}$, S.D. 423.4, range 54.2$1738,4)$ was significantly higher than mean level in inactive JIA patients $(304.77 \mathrm{pg} / \mathrm{mL}$, S.D. 170.2 , range 76.3-747.6, $\mathrm{p}<0.001)$ or in healthy control $(155.04$ $\mathrm{pg} / \mathrm{mL}$, S.D. 62.5, range 32.5-256.9, $\mathrm{p}<0.001$ ) (Fig. 3).

In all (active and inactive) JIA patients serum levels of sICAM-1 and VEGF presented significant positive correlation with ESR values: $r=0.33, p<0.05$ and $\mathrm{r}=0.66, \mathrm{p}<0.001$ respectively; PLT: $\mathrm{r}=0.32, \mathrm{p}<0.05$ and $\mathrm{r}=0.38, \mathrm{p}<0.01$, respectively; and CRP: $\mathrm{r}=0.31$, $\mathrm{p}<0.05$ and $\mathrm{r}=0.75, \mathrm{p}<0.001$, respectively. 
Interestingly, mean serum concentration of sICAM1 in the subgroup of JIA patients with abnormal capillaroscopic findings (dilated subpapillary venous plexus and/or irregular loops) was higher: 354.84 $\mathrm{ng} / \mathrm{mL}$ (S.D. 100.4, range 240.9-402.1) compared with the subgroup of JIA patients wihout naifold capilaroscopy abnormalities (267.29 ng/mL (S.D. 80.7, range 174.5-402.2) $(\mathrm{p}<0.01)$. Similarly, significant statistical differences were found beetwen mean serum VEGF levels in the subgroup of JIA patients with abnormal capillaroscopy compared with the subgroup of JIA patients wihout naifold capilaroscopy abnormalities: $533.4 \mathrm{pg} / \mathrm{mL}$ (S.D. 360.6, range 99.5-1263.9) vs. 464.3 $\mathrm{pg} / \mathrm{mL}$ (S.D. 423.1, range 119.7-1738.3) $(\mathrm{p}<0.05)$.

\section{Discussion}

We found that there are significant structural abnormalities in the microcirculation of children with JIA which comprise lengthening and widening of capillary loops, frequent presence of abnormal capillaries and/or the presence of widen subpapillary venular plexus.

So far two reports only have addressed capillaroscopy assessment of microcirculation in a separate group of JIA patients [21,22]. Unlike in our study, Dolezalova et al. did not reveal any significant differences in the capillary density, capillary width or the frequency of abnormal findings between a group of 15 JIA patients and healthy controls [21]. In the second study, involving 55 children with JIA, the capillary number, size, shape and arrangements were found to be similar to healthy controls, also [22].

The reason of discrepancies between these two studies and our findings are not readily apparent. It could be speculated that the proportion of patients with active disease might contribute to this disagreement. Indeed, in the study by Ingnegnoli et al. none of the patients was in acute phase of disease while in our group $58 \%$ of patients had active disease [22]. Although no direct significant association between capillaroscopic findings and disease activity was observed in our study, this could be due to the fact that the majority of our patients were receiving DMARDs including anticytokine therapy which might alleviate systemic inflammation. Intriguingly, in our group the presence of microvascular abnormalities was associated with significantly higher levels of serum sICAM and VEGF which are considered to reflect endothelial dysfunction in chronic inflammatory diseases such as collagen diseases. To the best of our knowledge this is the first report investigating relationships between structural abnormalities in microcirculation of children with JIA and the levels of biomarkers of endothelial injury. Our observations are in agreement with the studies performed in adult RA patients in whom significantly higher levels of sICAM1 were found in RA patients with clinical signs of sys- temic vasculitis as compared with those RA patients without clinically apparent vascular involvement. Although no significant correlation between sICAM-1 levels and the capillaroscopy findings were found, in the majority $(75 \%)$ of the RA patients with severe vascular changes in capillaroscopy, sICAM-1 levels exceeded normal cut off value [23].

This observations appear particularly interesting in view of many similarities that have recently emerged between the paradigm of inflammation in the pathogenesis of atherosclerosis and the well-established mechanisms of inflammation in the pathogenesis of systemic inflammatory joint diseases including/such as RA or JIA [24-29].

As mentioned above we did not find direct associations between activity of JIA and capillaroscopic abnormalities. However, sICAM-1 and VEGF showed weak, but significant correlations with laboratory acute phase parameters suggesting indirect relationships between capillary impairment and activity of systemic inflammation.

In summary we showed that there are significant changes in the microcirculation of children with JIA and that these structural abnormalities are associated with increased levels of biomarkers of endothelial injury. It could therefore be speculated that capillaroscopic abnormalities might reflect endothelial dysfunction which is considered to be a key event in the development of atherosclerosis in JIA patients. Whether capillaroscopic assessment might indeed be useful in early identification of children being at higher risk of development of atherosclerosis requires further prospective studies.

\section{References}

[ 1] Bloom BJ, Nelson SM, Eisenberg D, Alario AJ. Soluble intercellular adhesion molecule-1 and E-selectin as markers of disease activity and endothelial activation in juvenile idiopathic arthritis. $J$ Rheumatol. 2005;32(2):366-72.

[2] Klimiuk PA, Sierakowski S, Latosiewicz R, Cylwik JP, Cylwik B, Skowronski J, Chwiecko J. Soluble adhesion molecules (ICAM-1, VCAM-1, and E-selectin) and vascular endothelial growth factor (VEGF) in patients with distinct variants of rheumatoid synovitis. Ann Rheum Dis. 2002; 61(9):804-9.

[3] Hansel S, Lassing G, Pistrosch S. Endothelial dysfunction in young patients with long term rheumatoid arthritis and low disease activity. Atheroscler. 2003;170:177-80.

[4] Dessein PH, Joffe BI, Singh S. Biomarkers of endothelial dysfunction, cardiovascular risk factors and atherosclerosis in rheumatoid arthritis. Arthritis Res Ther. 2005;7(3):634-643.

[5] Grover S, Sinha RP, Singh U et al. Subclinical atherosclerosis in rheumatoid arthritis in India. $J$ Rheumatol. 2006;33: 244-47.

[6] Wallberg-Jonsson S, Cvetkovic JT, Sundqvist KG, Lefvert AK, Rantapää-Dahlqvist S. Activation of the immune system and inflammatory activity in relation to markers of atherothrombotic disease and atherosclerosis in rheumatoid arthritis. J Rheumatol. 2002;29(5):875-82. 
[7] Wang L, Feng G. Rheumatoid arthritis increased the risk of coronary hart disease via vascular endothelial injures. Med Hypotheses. 2004;63:442-45.

[8] Pizzorini C, Sulli A, Craviotto C, Tuccio M, Seriolo B, Cutolo M. Diagnostic perspectives in the rheumatologic vasculitis: the role of video-capillaroscopy. Reumatismo. 2002;54(2):99104.

[9] Anderson M E, Alle P D, Moore T, Hillier V, Taylor C J, Herrick A L. Computerized nailfold video capillaroscopy--a new tool for assessment of Raynaud's phenomenon. J Rheumatol. 2005;32(5):841-48

[10] Cutolo M, Sulli A, Secchi M E, Paolino S, Pizzorini C. Nailfold capillaroscopy is useful for the diagnosis and follow-up of autoimmune rheumatic diseases. A future tool for the analysis of microvascular heart involvement? Rheumatol (Oxford). 2006;45(Suppl 4):43-46.

[11] Cortes S, Cutolo M. Capillaroscopic patterns in rheumatic diseases. Acta Reumatol Port. 2007r;32(1):29-36.

[12] Cutolo M, Pizzorni C, Sulli A. Nailfold video-capillaroscopy in systemic sclerosis. J Rheumatol. 2004;63(6):457-62.

[13] Scardina GA, Messina P. Microvascular abnormalities in patients with rheumatoid arthritis. Ann Anat. 2006;188(5): 425-29.

[14] Silver RM, Maricq HR. Childhood dermatomyositis: serial microvascular studies. Pediatrics. 1989;83:278-283.

[15] Nascif AKS, Terreri MT, Len CA et al. Inflammatory myopathies in childhood: correlation between nailfold capillaroscopy findings and clinical and laboratory data. J Pediatr. (Rio J) 2006;82:40-45.

[16] Dolezalová P, Telekesová P, Nemcová D, Belorová J, Kvasnicka J, Hoza J. Laboratory indicators of endothelial involvement in rheumatic diseases associated with vasculitis in children. Cas Lek Cesk. 2003;142(10):615-9.

[17] Pizzorini C, Sulli A, Craviotto C, Tuccio M, Seriolo B, Cutolo M. Diagnostic perspectives in the rheumatologic vasculitis: the role of video-capillaroscopy. Reumatismo. 2002;54(2):99104.

[18] Chung CP, Avalos I, Raggi P, Stein CM. Atherosclerosis and inflammation: insights from rheumatoid arthritis. Clin Rheumatol. 2007;26(8):1228-33.
[19] Petty RE, Southwood TR, Baum J et al. Revision of the proposed classification criteria for juvenile idiopathic arthritis: Durban, 1997. J Rheumatol. 2002;29:1774-9.

[20] Vignola S, Picco P, Falcini F, Sabatini F, Buoncompagni A, Gattorno M. Serum and synovial fluid concentration of vascular endothelial growth factor in juvenile idiopathic arthritides. Rheumatol (Oxford). 2002;41(6):691-6.

[21] Dolezalova P, Young SP, Bacon PA, Southwood TR. Nailfold capillary microscopy in healthy children and childhood rheumatic diseases: a prospective single blind observational study. Ann Rheum Dis. 2003;62:444-49.

[22] Ingegnoli F, Zeni S, Gerloni V, Fantini F. Capillaroscopic observations in childhood rheumatic diseases and healthy controls. Clin Exp Rheumatol. 2005;23(6):905-11.

[23] Kuryliszyn-Moskal A, Bernacka K, Klimiuk PA. Circulating intercellular adhesion molecule 1 in rheumatoid arthritis relationship to systemic vasculitis and microvascular injury in nailfold capillary microscopy. Clin Rheumatol. 1996;15(4): $367-73$.

[24] Wallberg-Jonsson S, Ohman M, Rantapää-Dahlqvist S. Which factors are related to the presence of atherosclerosis in rheumatoid arthritis? Scand J Rheumatol. 2004;33(6):373-9.

[25] Szucs G, Tímár O, Szekanecz Z, Dér H, Kerekes G, Szamosi S, Shoenfeld Y, Szegedi G, Soltész P. Endothelial dysfunction precedes atherosclerosis in systemic sclerosis--relevance for prevention of vascular complications. Rheumatology (Oxford). 2007;46(5):759-62.

[26] Löwenhoff T, Głuszko P. Atherosclerosis and rheumatoid arthritis. Przegl Lek. 2005;62(12):1506-9.

[27] Hürlimann D, Enseleit F, Ruschitzka F. Rheumatoid arthritis, inflammation, and atherosclerosis. Herz. 2004;29(8):760-8.

[28] Bonetti PO, Lerman LO, Lerman A. Endothelial dysfunction: a marker of atherosclerotic risk. Arterioscler Thromb Vasc Biol. 2003;23(2):168-175.

[29] Pietrewicz E, Urban M. Early atherosclerosis changes in children with juvenile idiopathic arthritis. Pol Merk Lek. 2007; 22(129):211-4.

Submitted: 27 July, 2008 Accepted after reviews: 28 September 2008 